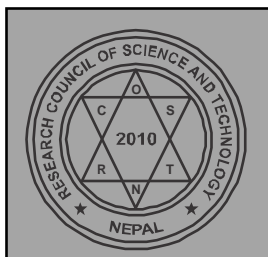

\title{
BIBECHANA
}

A Multidisciplinary Journal of Science, Technology and Mathematics

ISSN 2091-0762 (online)

Journal homepage: http://nepjol.info/index.php/BIBECHANA

\section{Some special characterisations of Fredholm operators in Banach space}

\author{
Mahendra Shahi \\ Department. of Mathematics, M.M.A.M. Campus, Biratnagar \\ Tribhuvan University, Nepal \\ E-mail: mshahi11@hotmail.com
}

Accepted for publication: February 06, 2014

\begin{abstract}
A bounded linear operator which has a finite index and which is defined on a Banach space is often referred to in the literature as a Fredholm operator. Fredholm operators are important for a variety of reasons, one being the role that their index plays in global analysis. The aim of this paper is to prove the spectral theorem for compact operators in refined form and to describe some properties of the essential spectrum of general bounded operators by the use of the theorem of Fredholm operators. For this, we have analysed the Fredholm operator which is defined in a Banach space for some special characterisations.
\end{abstract}

(C) 2014 RCOST: All rights reserved.

\section{Keywords: Bounded linear operator; Compact operator; Fredholm operator; Banach space.}

\section{Introduction}

An operator K defined by a kernel $\mathrm{k}$ is called a Fredholm type operator. The name goes back to Swede, E. Ivar Fredholm who developed a comprehensive theory for integral equations of second kind at the beginning of the twentieth century [4].

Let $\mathrm{X}=\mathrm{Y}=\mathrm{C}[\mathrm{a}, \mathrm{b}]$ be a Banach Space.Let $\mathrm{k}(\mathrm{s}, \mathrm{t})$ be defined for $\mathrm{a} \leq \mathrm{s} \leq \mathrm{band} \mathrm{a} \leq \mathrm{t} \leq \mathrm{b}$. Then for each $\mathrm{x} \in \mathrm{X}$ the Riemann integral

$$
\int_{a}^{b} k(s, t) x(t) d t
$$

exists and defines a continuous function of $s$ on $[a, b]$.

The integral (1) defines a linear operator $\mathrm{K}$ on $\mathrm{X}$ into $\mathrm{X}$. If we take $\mathrm{Kx}=\mathrm{y}$ to mean

$$
y(s)=\int_{a}^{b} k(s, t) x(t) d t
$$

the equation(2) is known as Fredholm type integral equation of the first kind [7].

Another operator $\mathrm{T}$ is obtained by defining

to mean

$$
\mathrm{Tx}=\mathrm{y}
$$




$$
y(s)=x(s)-\int_{a}^{b} k(s, t) x(t) d t
$$

Here $\mathrm{k}(\mathrm{s}, \mathrm{t})$ is a continuous function on $[\mathrm{a}, \mathrm{b}] \times[\mathrm{a}, \mathrm{b}]$ and is called the kernel of the integral equation. $\mathrm{y}(\mathrm{s})$ is continuous on $[\mathrm{a}, \mathrm{b}]$ and therefore $\mathrm{y} \in \mathrm{C}[\mathrm{a}, \mathrm{b}]$. The equation(3) is known as Fredholm type integral equation of the second kind [5].

Equations of this sort are of great importance. The application of this operator plays a vital role in the theory of boundary value problems in differential equations. Fredholm studied Fredholm type integral equations of the second kind, which gave rise to such operators. In view of the development of the theory of Fredholm operators the following definitions are frequently used.

\section{Definitions}

\section{Fredholm operator}

(i) A closed linear operator which has a finite index is called a Fredholm operator.

(ii) Let $X$ and $Y$ are Banach spaces. A linear operator $T$ from $X$ to $Y$ is called a Fredholmoperator if

i. $\quad T$ is closed.

ii. The domain of $T$ is dense in $X$.

iii. $\quad \alpha(T)$, the dimension of the null space $N(T)$ of $T$ is finite.

iv. The range of $T$ is closed in $y$

v. $B(T)$, The co-dimension of $R(T)$ in $Y$ is finite.

The terminology stems from the classical theory of integral equations. Special types of Fredholm operators were considered by many authors since that time but systematic treatment were not given until the work of Atkimon [1], Gohberg [4] and yood [9]. A general account of the history of the theory is given by [a]Gohberg Krein [3] and (b) Kato [5]. For a good general account of the theory can be found in the book written by Gohberg [4].

\section{Definition(3)}

Let B \& $\mathrm{C}$ be two Banach spaces. A bounded linear operator A: B $\rightarrow \mathrm{C}$ is defined to be a linear map for which the norm

is finite.

$$
\|\mathrm{A}\|:=\sup \{\| \text { Af }\|:\| \mathrm{f} \| \leq 1\}
$$

\section{Definition(4)}

Let $\mathrm{X}$ and $\mathrm{y}$ be normed linear spaces. Suppose $\mathrm{T}$ is a linear operator with domain $\mathrm{X}$ and range in $\mathrm{y}$. We say that $T$ is compact if for each bounded sequence $\{x n\}$ in $X$, the sequence $\{T x n\}$ contains a sub sequence converging to some limit in $\mathrm{y}$. A compact operator is also called completely continuous.

\section{Lemma (1)}

If $A$ is a compact operator on $B$, then $(\lambda I-A)$ is Fredholm for all $\lambda \neq 0$.

\section{Proof:}

We first prove that $\mathcal{L} \square \operatorname{Ker}(\lambda \mathrm{I}-\mathrm{A})$ is finite dimensional by contradiction. If this were not the case there would exist an infinite sequence $x_{n} \in \mathcal{L}$ such that $\left\|x_{n}\right\|=1$ and $\left\|x_{m}-x_{n}\right\| \geq 1 / 2$ for all distinct $m$ and $\mathrm{n}$. Since $\mathrm{Axn}=\lambda \mathrm{xn}$ and $\lambda \neq 0$, we could conclude that Axn has no convergent subsequence. We can write $\mathrm{B}=\mathcal{L}+\mathrm{M}$, where $\mathcal{L} \cap \mathrm{M}=\{0\}$ and $\mathrm{M}$ is a closed linear subspace on which $(\lambda \mathrm{I}-\mathrm{A})$ is one-one. We next prove that $\mathcal{R}:=\operatorname{Ran}(\lambda I-A)$ is closed. If $g_{n} \in \mathcal{R}$ and $\left\|g_{n}-g\right\| \rightarrow 0$, then there exist $f_{\mathrm{n}} \in M$ such that $g_{\mathrm{n}}=(\lambda I-A) f_{\mathrm{n}}$. If $\left\|\mathrm{f}_{\mathrm{n}}\right\|$ is not a bounded sequence then by passing to a subsequence (without 
change of notation) we may assume that $\left\|\mathrm{f}_{\mathrm{n}}\right\| \rightarrow \infty$ as $\mathrm{n} \rightarrow \infty$. Putting $\mathrm{h}_{\mathrm{n}}:=f_{n} /\left\|f_{n}\right\|$ we have $\left\|h_{n}\right\|=1$ and $k_{n}:=(\lambda I-A) h_{n} \rightarrow 0$. The compactness of $A$ implies that $h_{n}=\lambda^{-1}\left(A h_{n}+k_{n}\right)$ has a convergent subsequence. Passing to this subsequence we have $h_{n} \rightarrow h$ where $\|h\|=1, h \in \mathrm{M}$, and $h=\lambda^{-1} A h$. We conclude that $h \in \mathrm{M} \cap \mathcal{L}$. The contradiction implies that $\left\|f_{n}\right\|$ is a bounded sequence. Given this fact the compactness of $A$ implies that the sequence $f_{n}=\lambda^{-1}\left(A f_{n}+g_{n}\right)$ has a convergent subsequence. Passing to this subsequence we obtain $f_{n} \rightarrow f$ as $\mathrm{n} \rightarrow \infty$, sof $=\lambda^{-1}(A f+g)$, and $g=(\lambda I$ A) $f$. Therefore $\mathcal{R}$ is closed.

Since $\operatorname{Ran}(\lambda I-A)$ is closed, an application of the Hahn-Banach theorem implies that its codimension equals the dimension of $\operatorname{Ker}\left(\lambda I-A^{*}\right)$ in $\mathrm{B}^{*}$. But $A^{*}$ is compact, so this is finite by the first paragraph.

Our next theorem provides a second characterization of Fredholm operators.

\section{Theorem (1)}

Every Fredholm operator has closed range. The bounded operator $A: \mathrm{B} \rightarrow \mathrm{C}$ is Fredholm if and only if there is a bounded operator $B: \mathrm{C} \rightarrow \mathrm{B}$ such that both $(A B-I)$ and $(B A-I)$ are compact.

\section{Proof:}

If $A$ is Fredholm then $\mathrm{B}_{1}:=\operatorname{Ker}(A)$ is finite-dimensional and so has a complementary closed subspace $\mathrm{B}_{0}$ in $\mathrm{B}$. Moreover $A$ maps $\mathrm{B}_{0}$ one-one onto $\mathrm{Co}:=\operatorname{Ran}(A)$. If $\mathrm{C}_{1}$ is a complementary finite-dimensional subspace of $\mathrm{C}_{0}$ in $\mathrm{C}$ then the operator $X: \mathrm{B}_{0} \oplus \mathrm{C}_{1} \rightarrow \mathrm{C}$ defined by

$$
X(f \oplus v):=A f+v
$$

is bounded and invertible. We deduce by the inverse mapping theorem that $\mathrm{C}_{0}:=\mathrm{X}\left(\mathrm{B}_{0}\right)$ is closed. This completes the proof of the first statement of the theorem.

Still assuming that $A$ is Fredholm, put $B(\mathrm{~g} \oplus \mathrm{v}):=\left(\mathrm{A}_{0}\right)^{-1} \mathrm{~g}$ for all $g \in \mathrm{C}_{0}$ and $v \in \mathrm{C}_{1}$, where $A_{0}$ : $\mathrm{B}_{0} \rightarrow \mathrm{C}_{0}$ is the restriction of $A$ to $\mathrm{B}_{0}$. Then $B$ is a bounded operator from $\mathrm{C}$ to Band both of

$$
K_{1}:=A B-I, \quad K_{2}:=B A-I
$$

are finite rank and hence compact.

Conversely suppose that $A, B$ are bounded, $K_{1}, K_{2}$ are compact and (1) hold. Then

$$
\begin{aligned}
& \operatorname{Ker}(A) \subseteq \operatorname{Ker}\left(I+K_{2}\right), \\
& \operatorname{Ran}(A) \supseteq \operatorname{Ran}\left(I+K_{1}\right) .
\end{aligned}
$$

Since $\left(I+K_{1}\right)$ and $\left(I+K_{2}\right)$ are both Fredholm by Lemma (7.1), it follows that Amust be Fredholm.

The proof of Theorem (1) provides an important structure theorem for Fredholm operators.

\section{Theorem (2)}

If $A$ is a Fredholm operator then there exist decompositions $\mathrm{B}=\mathrm{B}_{0} \oplus \mathrm{B}_{1}$ and $\mathrm{C}=\mathrm{C}_{0} \oplus \mathrm{C}_{1}$ such that (i) $\quad \mathrm{B}_{0}$ and $\mathrm{C}_{0}$ are closed subspaces; 
(ii) $\quad \mathrm{B}_{1}$ and $\mathrm{C}_{1}$ are finite-dimensional subspaces;

(iii) $\quad \mathrm{B}_{1}=\operatorname{Ker}(A)$ and $\mathrm{C}_{0}=\operatorname{Ran}(A)$;

(iv) $\quad \operatorname{Index}(A)=\operatorname{dim}\left(\mathrm{B}_{1}\right)-\operatorname{dim}\left(\mathrm{C}_{1}\right)$;

(v) A has the matrix representation

$$
A=\left(\begin{array}{cc}
A_{0} & 0 \\
0 & 0
\end{array}\right)
$$

where $A_{0}: \mathrm{B}_{0} \rightarrow \mathrm{C}_{0}$ is one-one onto.

\section{Example (1)}

Let $A$ be a Fredholm operator on the Banach space B. Prove that if $\operatorname{Ker}(A)=\{0\}$ then $\operatorname{Ker}(A$ $\square I)=\{0\}$ for all small enough $\square$. It can be proved that if $\operatorname{Ran}(A)=$ Bthen $\quad \operatorname{Ran}(A-\square I)=\mathrm{B}$ for all small enough $\square$.

Before stating our next theorem we make some definitions. We say that $\lambda$ lies in the essential spectrum $\operatorname{EssSpec}(A)$ of a bounded operator $A$ if $(\lambda I-A)$ is not a Fredholm operator.

Since the set $\kappa(B)$ of all compact operators is a norm closed two-sided ideal in the Banach algebra $\mathcal{L}(\mathrm{B})$ of all bounded operators on $B$, the quotient algebra $\mathrm{C}:=\mathcal{L}(\mathrm{B}) / \kappa(\mathrm{B})$ is a Banach algebra with respect to the quotient norm

$$
\|\pi(A)\|:=\inf \{\|A+K\|: K \in \kappa(B)\}
$$

where $\pi: \mathcal{L}(\mathrm{B}) \rightarrow$ Cis the quotient map. The Calkin algebra $\mathrm{C}$ enables us to rewrite Theorem (1) is particularly in a simple form.

\section{Theorem (3)}

The bounded operator $A$ on $\mathrm{B}$ is Fredholm if and only if $\pi(A)$ is invertible in the Calkin algebra C. If $A \in$ $\mathcal{L}(\mathrm{B})$ then

$$
\operatorname{EsS\operatorname {Spec}}(A)=\operatorname{Spec}(\pi(A))
$$

\section{Proof :}

Both statements of the theorem are elementary consequences of Theorem (1).

\section{Corollary:}

If $A: \mathrm{B} \rightarrow \mathrm{B}$ is a Fredholm operator and $B:=A+K$ where $K$ is compact, then $B$ is a Fredholm operator and

$$
\operatorname{EsSSpec}(A)=\operatorname{EsSSpec}(B)
$$

\section{Theorem (4)}

If $A$ is a Fredholm operator on $\mathrm{B}$ then $A^{*}$ is Fredholm.

\section{Proof :}

Suppose that $A B=I+K_{l}$ and $B A=I+K_{2}$ where $K_{l}, K_{2}$ are compact. Then $B^{*} A^{*}=I+K_{I} *$ and $A * B^{*}=I+K_{2} *$. We deduce that $A *$ is Fredholm by applying Theorem (1). 


\section{Example (2):}

Prove directly from the definition that if $A_{1}$ and $A_{2}$ are both Fredholm operators then so is $A_{1} A_{2}$.

Note: If $\mathrm{B}_{1}=\mathrm{B}_{2}$ then this is an obvious consequence of Theorem (7.3), but there is an elementary direct proof.

\section{$\underline{\text { Theorem (5) }}$}

If $\mathrm{A}: \mathrm{B} \rightarrow \mathrm{C}$ is a Fredholm operator, then there exists $\mathrm{C}>0$ such that every bounded operator $\mathrm{X}$ satisfying $\|X-A\|<\in$ is also Fredholm with

\section{Proof:}

$$
\operatorname{index}(X)=\operatorname{index}(A) \text {. }
$$

We make use of the matrix representation of Theorem (2). If

$$
X=\left(\begin{array}{ll}
B & C \\
D & E
\end{array}\right)
$$

and $\|X-A\|<€$ then $\left\|B-A_{0}\right\|<\mathrm{c} \epsilon$, so $\mathrm{B}$ is invertible provided $\epsilon>0$ is small enough.

If $f \in B_{O}$ and $g \in B_{1}$ then $X(f \oplus g)=0$ if and only if

$$
\begin{aligned}
& B f+C g=0 \\
& D f+E g=0
\end{aligned}
$$

This reduces to

$$
\left(E-D B^{-1} C\right) g=0,
$$

where $\left(E-D^{-1} C\right) g: B_{1} \rightarrow C_{1}$, both of these spaces being finite-dimensional. We deduce that

$$
\operatorname{dim}(\operatorname{Ker}(X))=\operatorname{dim}\left(\operatorname{Ker}\left(E-D B^{-1} C\right)\right)
$$

for all small enough $C>0$. By applying a similar argument to

$$
X^{*}=\left(\begin{array}{ll}
B^{*} & D^{*} \\
C^{*} & E^{*}
\end{array}\right)
$$

we obtain

$$
\operatorname{dim}(\operatorname{Coker}(X))=\operatorname{dim}\left(\operatorname{Coker}\left(E-D B^{-1} C\right)\right)
$$

for all small enough $\varepsilon>0$.

Problem now implies that

$$
\left.\operatorname{index}(X)=\operatorname{index}\left(E-D B^{-1} C\right)\right)=\operatorname{dim}\left(\mathcal{B}_{1}\right)-\operatorname{dim}\left(C_{1}\right) \text {. }
$$

This formula establishes that index $(\mathrm{X})$ does not depend on $\mathrm{X}$, provided $\|X-A\|$ is small enough.

Theorem (5) establishes that the index is a homotopy invariant: if $t \rightarrow A_{t}$ is a norm continuous family of Fredholm operators then index $\left(\mathrm{A}_{t}\right)$ does not depend on $t$. In a Hilbert space context one can even identify the homotopy classes. 


\section{Conclusion}

Thus, we see that Fredholm operators are important for variety of reasons, one being the role that their index plays in global analysis. The dimension of null space $\mathrm{N}(\mathrm{T})$ and the co-dimension of $\mathrm{R}(\mathrm{T})$ of the operator $\mathrm{T}$ are finite and it is closed and range of the operator is also closed. The application of this operator plays a vital role in the theory of boundary value problems in differential equations.

\section{References}

[1]F-V Atkinson, Acta Sci. Math., 15 (1953) 38.

[2] Bovenbek Boss, J. Phillips, Canada. J. Math., 57 (2005) 225.

[3]I.C. Gohberb, M. G. Krein, Amer. Math. Soc. Transl., 13 (1960)185.

[4] S. Goldberg, Unbounded linear operators, Mc Graw Hill, New York, 1966.

[5] T Kato, Perturbation theory for linear operators, Springer, 1996.

[6] G. Kothe, Topological Vector Spaces, Vol. 1.and Vol. II, 1979.

[7] A.E. Taylor, D. C. Lay, Introduction Functional Analysis, 2nd Ed. Wiley, New York, 1980.

[8]A.E. Taylor, Theorems on ascent, descent, nullity and defect of linear operators, Math. Ann., 163 (1966) 18.

[9]B. Yood, Properties of linear transformations: Preserved under addition of a completely continuous trans. Duke Math., J. 18. 